\title{
The Efficacy of Milk Removal in Lactating Women with Vacuum Breast Pumps Medela Symphony and Breast Pump with Compression Component Laktopuls
}

\author{
Nikolay P Alekseev ${ }^{1 *}$, Vladimir I Ilyin ${ }^{1}$, Troschkin $\mathrm{MM}^{2}$ and Uleziko VA ${ }^{2}$ \\ ${ }^{1}$ Department of General Physiology, Saint Petersburg State University, Russia \\ ${ }^{2}$ ZAO PK Medthechnika, Russia
}

*Corresponding author: Nikolay P Alekseev, Faculty of Biology, Department of General Physiology, Saint Petersburg State University, St. Petersburg, Russian Federation.

To Cite This Article: Nikolay P Alekseev. The Efficacy of Milk Removal in Lactating Women with Vacuum Breast Pumps Medela Symphony and Breast Pump with Compression Component Laktopuls. Am J Biomed Sci \& Res. 2019 - 5(5). AJBSR.MS.ID.000947. DOI: 10.34297/AJBSR.2019.05.000948.

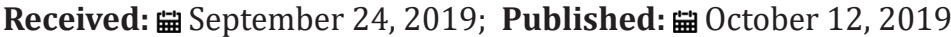

\begin{abstract}
Objective: To date, in the world has significantly increased the number women who use breast pump in the process of the breastfeeding. Many mothers preterm and term infants are partially or completely breast pump-dependent for weeks or months and mothers need a breast pump that is efficient and convenient. To ensure the continuity of lactation a woman should choose the apparatus in accordance with her mammary gland features. It is of fundamental interest to compare these features and the efficacy of breast milk expression by the two types of breast pumps vacuum pumps: The Medela Symphony and pumps with a compressive component: Lactopuls.
\end{abstract}

Material and Methods: Thirty lactating women of 19-38 years of age who volunteered to be included in this study were examined. Women had been lactating and breastfeeding normally for 5-8 days. Breast pump with vacuum and compressive components Lactopuls and beast pump with vacuum component Medela Symphony were used to express breast milk.

Results: It was found that the average values of the volume of milk expressed with the Lactopuls breast pump were $14 \%$ higher than the volume of milk expressed with the Medela Symphony breast pump. The Lactopuls breast pump empties the breasts more effectively than the Medela Symphony.

Conclusions: The use of breast pump Laktopuls with a vacuum and compression component has shown their higher efficacy compared with vacuum pump Medela Symphony

Keywords: Lactation; Breast Pump; Breast Pump Dependency; Efficacy

\section{Introduction}

To date, in the world has significantly increased the number of women who use breast pump in the process of the breastfeeding. The women who use breast pumps for correction of lactation can be divided into 3 groups [1]. The first group of mothers who feed healthy babies directly from the breast and a breast pump is needed for occasional or routine brief separations from the infant. These mothers are minimally breast pump dependent and the breastfeeding infant remains the regulator of lactation. Mothers who are partially breast pump dependent include those with late preterm, early term and the infants who consume small volumes of milk at the breast, slip off of the nipple frequently, fall asleep quickly after the feeding starts and do not awaken to feed at regular intervals. Third group is mothers who are completely breast pump dependent, such as mothers of extremely premature infants, the pump replaces the infant as the primary regulator of milk removal

and mammary gland stimulation and often does so for weeks or months after birth. Mothers whose term infants are unable to breastfeed (for example, craniofacial anomalies, hypotonia) or choose to provide exclusive mother milkby bottle are also completely breast pump dependent [1]. Besides this, irrespective of the ability of infants to stimulate the expression of milk, the mammary glands of different women have different anatomical and physiological characteristics, as well as the ability to form milk ejection and milk secretion reflexes. For example, if the breast nipple duct is thin, milk expression is slowed. Also, the formation of the milk ejection and milk secretion reflexes might require more intense stimulation 
of the areola receptors. Therefore, breast pumps must meet specific biomechanical requirements. They should:

a. Effectively mechanically stimulate mechanoreceptors in the areola of the mammary gland for the formation of the milk ejection and milk secretion reflexes in lactating women

b. Effectively empty the breasts,

c. Not damage the mammary gland not to cause pain in women during milk expression.

In Russia, there are currently two types of breast pump used in medical practice. The first type (Ameda, Medela, AVENT) applies only vacuum stimuli, while the second type of pump (Lactopulse and Perun) applies both vacuum and compression stimuli to the areola and the nipple, similar to removal of milk by an infant[2-5] Therefore, to ensure the continuity of lactation a woman should choose the apparatus in accordance with her mammary gland features [1]. It is of fundamental interest to compare these features and the efficacy of breast milk expression by the two types of breast pumps: vacuum pumps and pumps with a compressive component. The Medela Symphony hospital-grade breast pump and the Lactopuls Hospital-Grade breast pump with vacuum and compressive components have been chosen for this study.

\section{Material and Methods}

The study was conducted at the postnatal unit of the D.O. Ott Institute of Obstetrics, Gynecology and Reproductology in St. Petersburg, Russian Federation. Thirty lactating women of 19-38 years of age who volunteered to be included in this study were examined. Women had been lactating and breastfeeding normally for 5-8 days. Twelve of them were primiparous breastfeeding mothers and eighteen were multiparous mothers. The informed consent of the women was obtained according to the Declaration of Helsinki. The study was approved by the Ethics Committee of the D.O. Ott Institute of Obstetrics, Gynecology and Reproductology, RAN, Russia.

Breast pumps Medela Symphony and Laktopuls comprises a control unit and a removable cone shaped cup that is placed on the mammary gland. The cone shaped cup Laktopuls is made from transparent elastic silicon rubber and cone shaped cup Medela Symphony is made from transparent hard plastic fits a mammary gland to transfer the milk flow from the breast into the milk collection containers. The examination was conducted as follows: first, the mammary gland was expressed for 10 minutes by Laktopuls or Medela Symphony. Then the same gland was expressed for 10 minutes by Medela Symphony or Laktopuls, respectively. The amplitude of vacuum stimuli by the Laktopuls was $152 \mathrm{~mm} \mathrm{Hg}$ during the whole period of milk removal. The amplitude of compression was set during the first minute after the beginning of milk removal so that the patient felt intensive, but not painful compression of the gland areola. The Medela Symphony was functioning in the "maintain programme" mode. The duration of the stimulus phase was 1-1.5-minute, removal phase -10 minutes. Vacuum amplitude was $152 \mathrm{~mm} \mathrm{Hg}$. It should be noted that the amplitude of $152 \mathrm{~mm} \mathrm{Hg}$ did not cause any painful sensations in patients

Milk output was measured with an accuracy of $0.5 \mathrm{ml}$ and presented in the form of histograms as a ratio in percentage between the expressed milk and the total milk output (Figure 1). The reliability of differences in the means is tested by the Student's t-test. Statistical significance was set as $\mathrm{p}<0.05$.

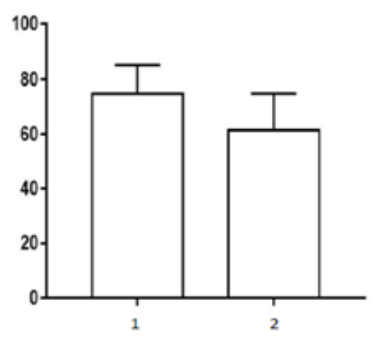

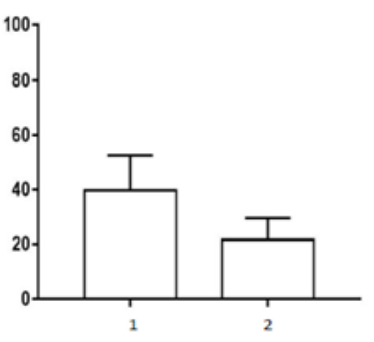

Figure 1: Volume of milk expressed by Laktopuls vs Medela Symphony.

A: Volume of milk expressed during first 10 minutes by Laktopuls (1) and Medela Symphony (2) $(p \leq 0.05)$

$\mathrm{B}$ : Volume of milk expressed during subsequent 10 minutes by Laktopuls (1) and Medela Symphony (2) ( $\mathrm{s} \leq 0.01)$.

\section{Results and Discussion}

It should be noted that all women who took part in the study have been using pumps on both breasts. Initial milk expression demonstrated that the volume of milk expressed by Laktopuls was $(75 \pm 10 \%)$ during 10 minutes (Figure $1 \mathrm{~A}, 1)$ which is $14 \%$ more in relation to the total milk output by Medela Symphony $(61 \pm 12 \%) p \leq 0.05)$ (Figure $1 \mathrm{~A}, 2)$. However, the difference between patients could be up to $60 \%$ in certain cases. A similar situation was observed during the subsequent 10 minutes of pumping. Laktopuls, after the first 10 minutes of milk expression by Medela Symphony, expressed on average $(38 \pm 12 \%)$ milk in relation to the total milk output (Figure 1B, 1), 16\% more than the Medela Symphony $(22 \pm 7 \%$ ) (Figure $1 \mathrm{~B}, 2)$ after initial milk expression by Laktopuls. The difference between patients could also amount to $60 \%$. Two patients, however, did not demonstrate any difference between the initial and subsequent milk expression by the two apparatus. As our observations revealed, the difference in the volume of expressed milk can be attributed to the morphology of the mammary glands and their functional state at milk ejection. In the case of exception, i.e. the expressed milk volume was the same, when using Laktopuls and Medela Symphony for ejection, milk drips from the gland quite freely and easily. Through a transparent cone shaped cup, it was seen that vacuum of an amplitude twice as 
little as recommended, i.e. 60-70 mm $\mathrm{Hg}$, caused milk expression. Additionally, after approximately 0.5 minute milk would start dripping intensively from the second gland, which indicates the formation of milk ejection reflexes. Concerning the other type of mammary glands, during the first minute of pumping, milk ejection was triggered only by applying the Laktopuls vacuum-compression stimuli breast pump. Furthermore, as reflex pressure increases in the alveolar duct system, milk outflow can be produced by vacuum only.

The results of the research conducted make it possible to conclude that milk removal by Laktopuls is more efficient that by Medela Symphony. It should be noted here that milk is accumulated in two compartments of glandular tissue. The first compartment includes thick and medium ducts, the second includes alveoli and thin ducts radiating from them. Most milk is accumulated in the second compartment. Milk expression from the first compartment depends mostly on the difference in pressure created by the air in a removable cone shaped cup and in the mammary gland ducts. In both apparatus the vacuum pressure was set at $152 \mathrm{~mm} \mathrm{Hg}$, but in the Laktopuls the ducts are additionally compressed, adding positive pressure to vacuum pressure. As a result, the difference between the intraductal pressure and the external environment increases and, as our research [6] proved, it results in an increase in the speed of milk expression.

In order to express milk from alveoli and thin ducts, milk ejection reflexes should be stimulated during the use of the Medela Symphony and Laktopuls breast pumps. The first structure for the formation of milk ejection reflexes is mostly the mechanoreceptors of the areola and the nipple. Morphological studies have revealed mechanoreceptors in the nipple and areola epidermis [7] which are also present in other areas of human skin [8]. No separate research on the functional characteristics of cutaneous mechanoreceptors of the mammary gland has been conducted. However, studies of the different types of cutaneous mechanoreceptors and their characteristics in other areas of human skin [8] demonstrated that an adequate stimulus would be stimulus by stretching and pressing (tactile stimuli). During milk expression by Laktopuls there is simultaneous stimulation of mechanoreceptors that respond to stretching and tactile stimuli. It intensifies the afferent impulses activity from nipple and areola mechanoreceptors, which results in a more efficient formation of milk ejection reflexes and positively affects the speed of milk expression [9].

During milk expression the Medela Symphony stimulates mostly the stretch mechanoreceptors. To intensify stimulation the Medela Symphony includes a special phase of stimulation ("maintain program" mode) [10]. Under this mode, in order to form the milk ejection reflexes the apparatus applies vacuum stimuli of lower amplitude and duration and of higher frequency (120 impulses/s) than stimuli for milk removal (47 impulses/s). It is believed to produce more effective stimulation of the mechanoreceptors of the mammary gland areola and nipple. Vacuum impulses are in the form of a half sinusoid with rising and falling phases of 250 ms [10]. It should be noted that stretch mechanoreceptors react only during the rising phase of skin stretching. There is no impulse activity during the falling phase. Vacuum milk ejection stimuli are trapezoidal [10]. The duration of rising and falling milk ejection stimuli is the same as the duration of special stimulation impulses. However, milk ejection stimuli have a plateau of $0.5 \mathrm{~s}$, during which the receptors also generate impulses. Therefore, vacuum stimuli for milk ejection would provide a higher stimulation of mechanoreceptors than special stimulation impulses. The effect of additional stimulation during milk removal by Medela Symphony might be considered insignificant for the formation of milk ejection reflexes. In fact, comparative tests [11] demonstrated that the Medela classic breast pump (without additional stimulation) was as efficient and effective as the Medela Symphony breast pump (with additional stimulation).

The efficacy of milk expression by the breast pump Medela Symphony can be increased by increasing vacuum pressure. A woman, however, might feel pain and this would affect milk ejection reflexes [12], which stimulate milk ejection from alveola and thin ducts into medium and wide ducts.

\section{Conclusions}

The use of breast pump Laktopuls with a vacuum and compression component has shown its higher efficacy compared with vacuum pump Medela Symphony. It suggests that the use of breast pump Laktopuls increase the efficacy of the breast pump due to the effective formation milk ejection reflex, and directly by an additional squeezing the milk from the breast ducts. Breast pump Laktopuls is more suitable to use for all types breast pump dependent women.

\section{References}

1. Meier PP, Patel A, Hoban R, Engstrom JL (2016) Which breast pump for which mother: an evidence-based approach to individualizing breast pump technology. J Perinatol 36(7): 493-499.

2. Ardran GM, Kemp FH, Lind J (1958) A cineradiographic study of breast feeding. Br J Radiol 31: 156-162.

3. Mizuno K, Ueda A (2001) Development of sucking behavior in infants with Down's syndrome. Acta Paediatr 90(12): 1384-1388.

4. Burton P, Deng J, McDonald D, Fewtrell MS (2013) Real-time 3D ultrasound imaging of infant tongue movements during breast-feeding. Early Hum Dev 89(9): 635-641.

5. Elad D, Kozlovsky P, Blum O, Zelicovich M, Ben Sira L, et al. (2014) Biomechanics of milk extraction during breast-feeding. Proc Natl Acad Sci USA 111(14): 5230-5235.

6. Alekseev NP, Ilyin VI (2016) The mechanics of breast pumping: compression stimuli increased milk ejection. Breastfeed Med 11: 370375 .

7. Miller MR, Kasahara M (1959) The cutaneous innervation of the human female Breast. Anat Rec 135: 153-167. 
8. Johansson RS, Vallbo AB (1983) Tactile sensory coding in the glabrous skin of the human hand. Trend Neusci 6: 27-32.

9. Alekseev NP, Ilyin VI, Yaroslavski VK, Omelyanjuk EV, Tkachenko NN, et al. (1998) Compression stimuli increase the efficacy of breast pump function. Eur J Obstet Gynecol Reprod Biol 77(2): 131-139.

10. Greter A, Larsson M (2003) Suction sequences for a breast pump. Patent USA.
11. Meier PP, Engstrom JL, Hurst NM, Zuleger JL, Jegier BJ, et al. (2008) A comparison of the efficiency, efficacy, comfort, and convenience of two hospital-grade electric breast pumps for mothers of very low birthweight infants. Breastfeed Med 3(3): 141-150.

12. Ueda T, Yokoyama Y, Irahara M, Aono T (1994) Influence of psychological stress on suckling-induced pulsatile oxytocin release. Obstet Gynecol 84(2): 259-262. 